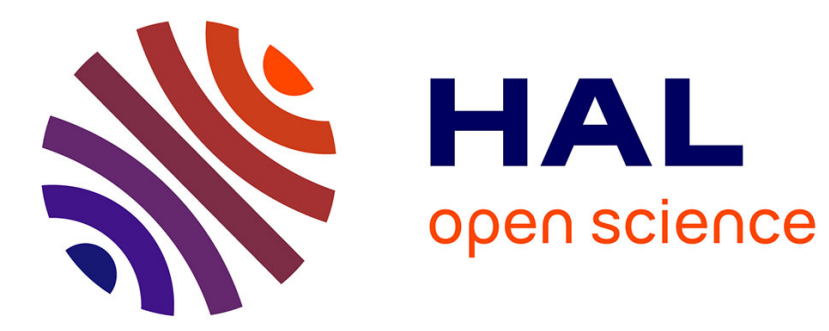

\title{
Random walks in two-dimensional complexes
}

\author{
Guy Fayolle, I.A. Ignatyuk, Vadim A. A. Malyshev, M.V. Menshikov
}

\section{To cite this version:}

Guy Fayolle, I.A. Ignatyuk, Vadim A. A. Malyshev, M.V. Menshikov. Random walks in twodimensional complexes. RR-1312, INRIA. 1990. inria-00075247

\section{HAL Id: inria-00075247 https://hal.inria.fr/inria-00075247}

Submitted on 24 May 2006

HAL is a multi-disciplinary open access archive for the deposit and dissemination of scientific research documents, whether they are published or not. The documents may come from teaching and research institutions in France or abroad, or from public or private research centers.
L'archive ouverte pluridisciplinaire HAL, est destinée au dépôt et à la diffusion de documents scientifiques de niveau recherche, publiés ou non, émanant des établissements d'enseignement et de recherche français ou étrangers, des laboratoires publics ou privés. 


\section{Rapports de Recherche}

UNITÉ DE RECHERCHE INRIA-ROCOUENCOURT
$\mathrm{N}^{\circ} 1312$

Programme 3

Réseaux et Systèmes Répartis

RANDOM WALKS IN TWO-DIMENSIONAL COMPLEXES
Institut National de Recherche en Informatique et en Automatique

Domaine de Voluceau Rocquencourt B.P.105 78153 Le Chesnay Cedex France Tél.:(1)39635511

\author{
Guy FAYOLLE \\ Irina IGNATYUK \\ Vadim MALYSHEV \\ Michael MENSHIKOV
}

Octobre 1990 


\title{
Marches aléatoires sur des simplexes bi-dimensionnels
}

\author{
Guy Fayolle*, Irina Ignatyuk ${ }^{* *}$, Vadim Malyshev**, Michael Menshikov**
}

\author{
April 1990
}

\section{Résumé}

Dans ce papier, nous étudions des marches aléatoires évoluant sur un simplexe formé d'un nombre fini de quarts de plan $\mathbf{Z}_{+}^{2}$, ayant des frontières communes. Sous des hypothèses d'homogénéité spatiale et de sauts bornés, on trouve les conditions explicites d'ergodicité et de transience du processus ainsi construit. La méthode, déjà utilisée dans des travaux précédents, est basée sur le raccordement idoine de fonctions de Lyapounov locales. Cette analyse est aussi d'importance théorique pour la classification de marches aléatoires dans $\mathbf{Z}_{+}^{4}$, ainsi que pour certains modèles de réseaux de files d'attente comportant des interactions.

* Adresse: INRIA, Domaine de Voluceau-Rocquencourt,

B.P.105 - 78153 LE CHESNAY - FRANCE.

Ad Adresse: Mechanico-Mathematical Faculty,

Department of probability ,

Moscow State University, Leninskie Gori,

119899 MOSCOW- USSR. 


\title{
Random walks in two-dimensional complexes
}

\author{
G. Fayolle*, I.A Ignatyuk**, V.A. Malyshev ${ }^{* *}$, M.V Menshikov ${ }^{* *}$
}

April 1990

\begin{abstract}
In this paper, we analyze random walks evolving in a region formed by a finite number of quarter planes $\mathbf{z}_{+}^{2}$ having some boundaries in common.Under assumptions involving essentially space homogeneity and boundedness of the jumps, it is possible to find the conditions for ergodicity and transience. The method implies a pasting of local Lyapounov functions, as done in earlier papers. This study is of theoretical importance for the classification of random walks in $\mathbf{Z}_{+}^{4}$. It also can represent some queueing networks with interactions.
\end{abstract}

* Postal address : INRIA, Domaine de Voluceau-Rocquencourt, B.P.105 - 78153 LE CHESNAY - FRANCE.

** Postal address : Mechanico-Mathematical Faculty, Department of probability , Moscow State University, Leninskie Gori, 119899 MOSCOW- USSR 


\title{
Random walks in two dimensional complexes
}

\author{
G.Fayolle, I.A. Ignatyuk, V.A. Malyshev, M.V. Menshikov
}

A 2-dimensional complex is a union of a finite number of quarter planes $\mathbf{Z}_{+}^{2}$ having some boundaries in common. An example can be the union of all 2-dimensional faces of $\mathbf{Z}_{+}^{N}$. We consider maximally homogeneous random walks on such complexes and obtain necessary and sufficient conditions for ergodicity and transience up to some "non zero" assumptions which are of measure 1 in the parameter space.

The main reason for studying this problem is that it is the step in advance comparatively to the paper [1] towards obtaining classification of maximally homogeneous random walks in $\mathbf{Z}_{+}^{N}$. In [1] the vector field was constructed in terms of which a complete classification was obtained for $N=2,3$ and sufficient conditions for ergodicity and transience were derived for $N>3$. One of the main features of the vector ficd in question that it was deterministic. Our advancement here is that certain vector fields appear which are deterministic inside 2-dimensional faces, but give rise to random scattering on 1-dimensional faces. This is the new phenomena which is common also for $\mathbf{Z}_{+}^{N}$. We give explicit solution to our problem : in fact it reduces to finding stationary probabilities for finite Markov chain with $n$ states, where $n$ is the number of 2-dimensional faces in the simplex considered.

The paper is organized as follows.

After the main definitions and preliminary results in sections 1,2 we formulate the main theorem 3 in section 3 . The proof of the ergodicity conditions using the method of pasting together local Lyapounov functions similar to used in [1] is given in section 4. Transience is proved in section 5 by using a simpler method for constructing more global Lyapounov functions. Possible generalizations are briefly described in section 6 . 


\section{Definitions and preliminary results}

We call a two-dimensional complex $\tilde{T}$ any union of finite number of copies of $\mathbf{R}_{+}^{2}$ :

$$
\left(\mathbf{R}_{+}^{2}\right)_{i}=\left\{\left(x_{1}, x_{2}\right)_{i}: x_{j} \geq 0\right\}, i=1, \ldots, n .
$$

We assume that all origins are identified, i.e. $(0,0)_{i} \stackrel{\text { def }}{=} \underline{0}$ for all $i$. Some pairs $\left(\tilde{\Lambda}_{j i}^{(1)}, \tilde{\Lambda}_{j_{1} i_{1}}^{(1)}\right)$ of its one dimensional faces.

$$
\tilde{\Lambda}_{1 i}^{(1)}=\left\{\left(x_{1}, 0\right)_{i}: x_{1}>0\right\}, \tilde{\Lambda}_{2 i}^{(1)}=\left\{\left(0, x_{2}\right)_{i}: x_{2}>0\right\}
$$

can be identified as well. This means that the points of $\tilde{\Lambda}_{j i}^{(1)}$ and $\tilde{\Lambda}_{j_{1} i_{1}}^{(1)}$ lying at the some distance from the origin are identified.

We shall consider discrete time homogeneous Markov chains $\mathcal{L}=\mathcal{L}_{T}$ with state space $T$, the integer points of $\tilde{T}$, i.e. the union of all

$$
\left(\mathbf{Z}_{+}^{2}\right)_{i}=\left\{\left(x_{i}, x_{2}\right)_{i}: x_{j} \geq 0 \text { integers }\right\} \subset\left(\mathbf{R}_{+}^{2}\right)_{i}
$$

taking into consideration the above identifications between them. We denote the inner part of a generic 2-dimensional face by $\tilde{\Lambda}^{(2)}$, e.g

$$
\tilde{\wedge}_{1}^{(2)}=\left\{\left(x_{1}, x_{2}\right)_{1}: x_{1} x_{2}>0\right\} \text {. }
$$

Introduce also $\pi^{(1)}, \pi^{(2)}$ - the closures of $\tilde{\Lambda}^{(1)}, \tilde{\Lambda}^{(2)}$, and $\wedge^{(1)}, \wedge^{(2)}$ - the sets of integer points of $\tilde{\Lambda}^{(1)}$ and $\tilde{\Lambda}^{(2)}$ respectively.

\section{Examples}

1. $\tilde{T}$ (or $T$ ) is called planar if any 1-dimensional face is identified with at most one other 1-dimensional face. This means that $\tilde{T}$ can be topologically imbedded into $\mathbf{R}^{2}$. The examples are :

(a) $\mathbf{Z}_{+}^{2}$,

(b) The union of 4 quadrants of $\mathbf{Z}^{2}$,

(c) the union of three 2-dimensional faces of $\mathbf{Z}_{+}^{3}$. 
2. The union of all $\frac{N(N-1)}{2} 2$-dimensional faces of $\mathbf{Z}_{+}^{N}, N \geq 4$, is the example of non-planar complex

3. $\tilde{T}$ is called strongly connected if it is not the union of two complexes $\tilde{T}_{1}$ and $\tilde{T}_{2}$ which have only $\underline{0}$ as a common point.

Let us note that in $\tilde{T}$ any line has a length. Whence, $\tilde{T}$ is the metric space endowed with the distance $\rho(\alpha, \beta)$, between $\alpha, \beta \in \tilde{T}$, equal to the minimal length of a line between them.

We assume that one-step transition probabilites $p_{\alpha \beta}(\alpha \rightarrow \beta)$ satisfy the following conditions :

\section{A1. Boundedness of jumps}

(a) $p_{\alpha \beta}=0$ if $\alpha, \beta$ do not belong to the same $\pi^{(2)}$;

(b) $p_{\alpha \beta}=0$ if $\rho(\alpha, \beta)>d$ for some fixed $d<\infty$;

(c) $p_{\alpha \beta}=0$ if at least one component of the vector $\beta-\alpha$ is less than -1 .

\section{A2. Simplest space homogeneity}

Let $\alpha, \alpha^{\prime}$ belong to the same (open) face $\wedge$ which can be 1 or 2 -dimensional. If $\wedge \subset \pi^{(2)}$ (i.e. $\Lambda=\Lambda^{(2)}$ or $\wedge$ is a one-dimensional face of $\wedge^{(2)}$ ) and, moreover,

$$
\beta^{\prime}-\alpha^{\prime}=\beta-\alpha
$$

then

$$
p_{\alpha \beta}=p_{\alpha^{\prime} \beta^{\prime}} \stackrel{\text { def }}{=} p_{\beta-\alpha}^{\wedge} \text {. }
$$

Thus, our Markov chain is uniquely specified by a finite number of parameters $p_{\gamma}^{\wedge}$, with $\gamma \in \pi^{(2)}$ such that $\wedge \subset \pi^{(2)}$.

We also shall make some assumptions $0_{1}, \ldots, 0_{5}$, which we call "non-zero assumptions" and which exclude from our consideration some hypersurfaces in the parameters space (in particular they are of measure zero in the parameter space). Some of these assumptions are made just for economy of space-time but others are very essential. They will appear thereafter.

\section{Assumption $0_{1}$}

The Markov chain $\mathcal{L}_{T}$ is supposed to be irreducible and aperiodic. 
Then e.g., this chain is ergodic iff any of its strongly connected component is ergodic. Therefore, we shall consider only strongly connected complexes $T$. For any 2-dimensional face $\wedge$ and any $\alpha \in \wedge$, we define the vectors $M(\alpha)$, the one-step mean jumps from $\alpha$. They are all equal to

$$
M_{\wedge}=\sum_{\beta \in \bar{\lambda}}(\beta-\alpha) p_{\beta-\alpha}^{\wedge}=M(\alpha), \forall \alpha \in \wedge
$$

If $\alpha \in \wedge^{(1)} \equiv \wedge$ then we define $M_{\wedge}=M(\alpha)$ to be the collection of vectors $M_{\wedge, \wedge}$ and $M_{\wedge, \wedge^{(2)}}$, for all $\overline{\wedge^{2}} \supset \wedge$, where

$$
\begin{aligned}
M_{\wedge, \wedge} & =\sum_{\beta \in \bar{\Lambda}}(\beta-\alpha) p_{\beta-\alpha}^{\wedge}, \\
M_{\wedge, \wedge^{(2)}} & =\sum_{\beta \in \wedge^{(2)}}(\beta-\alpha) p_{\hat{\beta}-\alpha}^{\wedge} .
\end{aligned}
$$

If one can imbeed $\tilde{T}$ into $\mathbf{R}^{N}$ for some $N$, so that all $\wedge^{(2)}$ are orthogonal, then $M(\alpha)$ for $\alpha \in \wedge^{(1)}$ can be defined as the usual vector of mean jumps.

Theorem 1.1 If at least for one $\wedge=\wedge^{(2)}$ the vector $M_{\wedge}$ has both components positive then $\mathcal{L}_{T}$ is transient

\section{Assumption $0_{2}$}

For any $\wedge$ the vector $M_{\wedge}$ can have no zero component.

\section{Definition 1}

Let $\Lambda^{(1)}$ be 1 -dimensional face and $S\left(\Lambda^{(1)}\right)$ be the set of all 2-dimensional faces $\wedge^{(2)}$ such that $\wedge^{(1)} \subset \overline{\Lambda^{(2)}} ; S_{+}\left(\wedge^{(1)}\right) \subset S\left(\wedge^{(1)}\right)$ be the set of all $\wedge^{(2)}$ such that $M_{\Lambda^{(2)}}$ looks onto $\Lambda^{(1)}$, i.e. its component perpendicular to $\Lambda^{(1)}$ is negative. Accordingly, $S_{-}\left(\wedge^{(1)}\right)=S\left(\wedge^{(1)}\right)-S_{+}\left(\wedge^{(1)}\right)$. We call $\wedge^{(2)} \in S_{+}\left(\wedge^{(1)}\right)$ [resp. $S_{-}\left(\wedge^{(1)}\right)$ ] an ingoing [resp. outgoing] face for $\wedge^{(1)}$.

If $S_{+}\left(\wedge^{(1)}\right)=S\left(\wedge^{(1)}\right)$, then $\wedge^{(1)}$ is called ergodic.

\section{Definition 2}

Let us consider a 1-dimensional face $\wedge^{(1)}$ and a point $\alpha \in \Lambda^{(1)}$. For any 2-dimensional $\wedge \in S\left(\wedge^{(1)}\right)$, let us consider the half-line $C_{\wedge^{(1)}}^{\wedge}$ which belongs 
to $\pi$ and is perpendicular to $\wedge^{(1)}$ at the point $\alpha$. We call the following one dimensional complex

$$
H_{\wedge(1)}=\bigcup_{\wedge \in S\left(\wedge^{(1)}\right)} C_{\wedge^{(1)}}^{\wedge}
$$

a hedgehog. For different $\alpha \in \wedge^{(1)}$, these hedgehogs are congruent in the obvious sense. Let us consider the Markov chain $\mathcal{L}_{\Lambda^{(1)}}$, with set of states $H_{\wedge(1)}$ (we call it the induced Markov chain for $\wedge^{(1)}$ ) and one-step transition probabilities which are the following projections

$$
q_{\alpha \beta}^{\wedge^{(1)}}=\sum_{\beta^{\prime}} p_{\alpha \beta^{\prime}}, \alpha, \beta \in H_{\wedge(1)},
$$

where the summation is over all $\beta^{\prime}$, such that $\beta^{\prime}$ belongs to the same face as $\beta$ and (if this face is $\wedge=\wedge^{(2)}$ ) the straight line connecting $\beta$ and $\beta^{\prime}$ is perpendicular to $C_{\wedge_{(1)}}$. From the homogeneity conditions, it follows that the induced chain for $\wedge^{(1)}$ does not depend on the choice of $\alpha \in \wedge^{(1)}$.

\section{Assumption $0_{3}$}

For any $\wedge^{(1)}$, the induced chain $\mathcal{L}_{\wedge^{(1)}}$ is irreducible and aperiodic.

Then $\mathcal{L}_{\wedge^{(1)}}$ is ergodic iff $\wedge^{(1)}$ is ergodic. This explains the word. Let $\pi_{\Lambda^{(1)}}(h)$. be the stationary probabilities of $\mathcal{L}_{\Lambda^{(1)}}, h \in H_{\Lambda^{(1)}}$, in the ergodic case.

Let us define, for any ergodic, $\wedge^{(1)}$ a number $v_{\wedge^{(1)}}$ - the "second vector field" on 1-dimensional ergodic faces.

$$
v_{\wedge^{(1)}}=\sum_{h \in H_{\Lambda^{(1)}}} \pi_{\wedge^{(1)}}(h) P r_{\wedge^{(1)}} M(h),
$$

where $\operatorname{Pr}_{\wedge^{(1)}}$ means orthogonal projection of $M(h)$ onto $\wedge^{(1)}$. If $h \in \wedge^{(1)}$, this means

$$
\operatorname{Pr}_{\wedge^{(1)}} M(h)=M_{\wedge^{(1)} \wedge^{(1)}}+\sum_{\wedge^{(2)}} \operatorname{Pr}_{\wedge^{(1)}} M_{\wedge^{(1) \wedge^{(2)}}}
$$

Theorem 1.2 If $v_{\wedge(1)}>0$ for at least one ergodic $\wedge^{(1)}$, then $\mathcal{L}_{T}$ is transient. Assumption $0_{4}$ $v_{\wedge(1)} \neq 0$ for all $\wedge^{(1)}$.

Now we want to show that the sign of $v_{\wedge(1)}$ is easily calculated 


\section{Lemma 1.3}

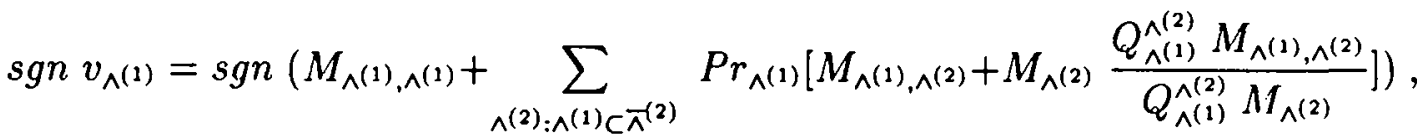

where $Q_{\wedge^{(1)}}^{(2)}$ is the projection of vector in $\wedge^{(2)}$ onto the axis of $\wedge^{(2)}$ other than $\wedge^{(1)}$.

\section{Random walks on hedgehogs}

For a given hedgehog $H_{\wedge^{(1)}}$, we call $C_{\Lambda^{(1)}}^{\wedge^{(2)}}$ its bristle. A bristle $C_{\Lambda^{(1)}}^{\wedge^{(2)}}$ is called ingoing if $\wedge^{(2)}$ is ingoing, i.e. if the following number (representing the mean jump along the bristle)

$$
m_{\wedge(2)}=\sum_{h^{\prime}}\left(h^{\prime}-h\right) q_{h h^{\prime}}^{(1)},
$$

which does not depend on the position of $h \in C_{\Lambda^{(1)}}^{\wedge^{(2)}}$, is negative.

When $\wedge^{(1)}$ is not ergodic, we shall define a "scattering" probability $p_{s c}\left(\wedge^{(1)}, \wedge^{(2)}\right)$, for $\wedge^{(2)} \in S_{-}\left(\wedge^{(1)}\right)$, which is the probability that the particle will go to infinity along $C_{\Lambda^{(1)}}^{\wedge^{(2)}}$. Under our simplest homogeneity assumptions, this definition does not depend on the initial position, provided that this latter is either at the origin of the hedgehog or on some ingoing bristle. Thus, we can assume that it is at the origin $0 \in H_{\wedge(1)}$.

\section{Computation of the scattering probabilities}

Let us fix $\wedge^{(1)}, \wedge^{(2)}$ and put $q_{h h^{\prime}}=q_{h h^{\prime}}^{(1)}$. It is clear that

$$
p_{s c}\left(\wedge^{(1)}, \wedge^{(2)}\right)=\frac{\sum_{h \in C_{\Lambda(1)}^{\wedge(2)}} q_{0 h} p(h)}{\sum_{0 \neq h \in H_{\wedge(1)}} q_{0 h} p(h)}
$$

where $p(h) \equiv p_{\wedge^{(2)}}(h)$ is the probability that, starting from $h$, the particle on the hedgehog will never return to 0. Formula (2.2) follows from the fact that

$$
p_{s c}\left(\wedge^{(1)}, \wedge^{(2)}\right)=\text { const } \sum_{h \in C_{\wedge^{(1)}}^{(2)}} q_{0 h} p(h)
$$


where const does not depend on the outgoing $\wedge^{(2)}$. We shall show now that

$$
p(h)=1-(1-\gamma)^{h},
$$

where $\gamma=p(1)$ is the unique root inside the unit disc of the equation

$$
(1-\gamma)^{h}=\sum_{h^{\prime}} q_{h h^{\prime}}(1-\gamma)^{h^{\prime}}
$$

with $h \in C_{\Lambda^{(1)}}^{\Lambda^{(2)}} /\{0\}$ (e.g. we can take $h=1$ ), and $h^{\prime} \in C_{\Lambda^{(1)}}^{\Lambda^{(2)}}$.

The proof is easily obtained from the recursive relationship

$$
p(h+1)=p(1)+[1-p(1)] p(h)
$$

and standard generating function method.

\section{Proof of lemma 1.3}

Let $H_{\wedge^{(1)}}$ be a hedgehog such that all $m_{\wedge^{(2)}}$ are negative, for all its bristles $C_{\wedge(1)}^{\wedge(2)}$.

Let

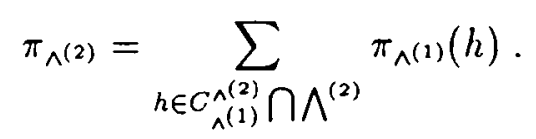

We claim that

$$
\frac{\pi_{\Lambda^{(2)}}}{\pi_{0}}=\frac{Q_{\Lambda^{(1)}}^{\wedge^{(2)}} M_{\wedge^{(1)}, \wedge^{(2)}}}{Q_{\Lambda^{(1)}}^{\Lambda^{(2)}} M_{\Lambda^{(2)}}} .
$$

To prove this, we note first that, for computing the above quantity, it suffices to consider a modified random walk on the bristle $C_{\wedge^{(1)}}^{\wedge^{(2)}}$, i.e. on $\mathbf{Z}_{+}^{1}$, after slightly "updating" the transition probabilities. More exactly, we define $\tilde{q}_{h h^{\prime}}=q_{h h^{\prime}}$, for all $h, h^{\prime} \in C_{\Lambda^{(1)}}^{\wedge^{(2)}}$, except for $\tilde{q}_{00}$ which is taken equal to

$$
\tilde{q}_{00}=1-\sum_{0 \neq h^{\prime} \in C_{\wedge^{\wedge}(1)}^{(1)}} \tilde{q}_{0 h^{\prime}} .
$$

Then $\frac{\pi_{\Lambda^{(2)}}}{\pi_{0}}$ does not depend on this modification and its value in the case of $\mathbf{Z}_{+}^{1}$ is a well-known result, yielding in particular exact ergodicity conditions for random walks in $\mathbf{Z}_{+}^{2}$. [The point is that, due to the homogeneity, it is not necessary to compute the exact values of the $\pi_{\wedge(1)}(h)^{\prime} s$ : only the drifts are needed]. The proof is concluded. 


\section{Formulation of the main result}

\section{Definition 3}

For given $T$ and $\mathcal{L}_{T}$, we define the following associated Markov chain $\mathcal{M}$ having a finite number of states $n=|T|$, equal to the number of 2-dimensional faces of $T$. [It is thus natural to denote these states by $\wedge^{(2)}$ ]. The one-step transition probabilities $p\left(\wedge_{i}^{(2)}, \wedge_{j}^{(2)}\right)$ of $\mathcal{M}$ are equal to

$$
p\left(\wedge_{i}^{(2)}, \wedge_{j}^{(2)}\right)=\left\{\begin{array}{l}
p_{s c}\left(\wedge^{(1)}, \wedge_{j}^{(2)}\right), \text { if } \wedge_{i}^{(2)} \in S_{+}\left(\wedge^{(1)}\right), \wedge_{j}^{(2)} \in S_{-}\left(\wedge^{(1)}\right) \\
1, \text { if } \wedge_{i}^{(2)}=\wedge_{j}^{(2)} \in S_{+}\left(\wedge^{(1)}\right) \text { for some ergodic } \wedge^{(1)} \\
0, \text { otherwise. }
\end{array}\right.
$$

We do not exclude that the associated chain be reducible or periodic. Let $\mathfrak{A}_{1}, \ldots, \mathfrak{A}_{k}$ be irreducible classes of essential states. Let us consider some class $\mathfrak{A}_{i}$ with $\left|\mathfrak{x}_{i}\right| \geq 2$. We define the following function $f$ on $\mathfrak{x}_{i}$ :

If $\wedge^{(2)} \in \mathfrak{A}_{i}$ and $\phi_{\Lambda^{(2)}}$ is the angle betwcen $M_{\Lambda^{(2)}}$ and the negative axis from which $M_{\wedge(2)}$ gocs away,

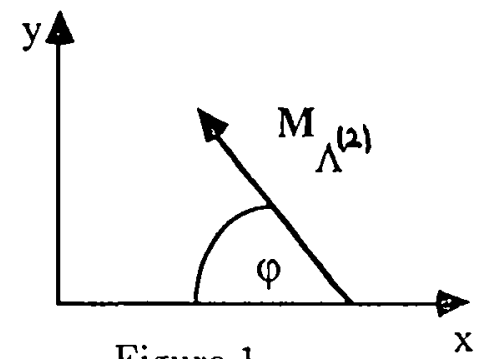

Figure 1

then we put

$$
f\left(\wedge^{(2)}\right)=\log \operatorname{tg} \phi_{\wedge(2)} .
$$

Heuristically, if we are c.g. On the $x$-axis of $\wedge^{(2)}$ at a point $(x, 0)$ and we move along the constant vector ficld $\Lambda_{\Lambda^{(2)}}$ to a point $(0, y)$ of the y-axis, then $\exp f\left(\wedge^{(2)}\right)$ represents the dilatation cocfficient $\alpha=\frac{y}{x}$.

- If $\mathfrak{A}_{i}$ is aperiodic and $\pi_{i}\left(\wedge^{(2)}\right)$ denotes its stationnary probabilities, we define

$$
M\left(\mathfrak{A}_{i}\right)=\sum_{\wedge^{(2)} \in \mathfrak{A}_{i}} \pi_{i}\left(\wedge^{(2)}\right) f\left(\wedge^{(2)}\right)
$$


- If $\mathfrak{A}_{i}$ is periodic, $\pi_{i}\left(\wedge^{(2)}\right)$ is then taken to be the stationary probability in the aperiodic subclass containing $\wedge^{(2)}$.

The vector $M\left(\mathfrak{a}_{i}\right)$ is defined by the same formula (3.1). Perhaps it will be more convenient to normalize it, multiplying by $N\left(\mathfrak{A}_{i}\right)^{-1}$ where $N\left(\mathfrak{A}_{i}\right)$ is the number of aperiodic subclasses in $\mathfrak{x}_{\boldsymbol{i}}$.

\section{Assumption $0_{5}$}

For all $i, M\left(\mathfrak{a}_{i}\right) \neq 0$

Our main result is the following

Theorem 3.1 Under the assumptions $0_{1}, \ldots, 0_{5}$ and if the assumptions of theorems 1 and 2 are not fulfilled, then $\mathcal{L}_{T}$ is ergodic iff, for any $\boldsymbol{x}_{i}$ with $\left|\mathfrak{A}_{i}\right| \geq 2$,

$$
M\left(\boldsymbol{a}_{i}\right)<0 .
$$

\section{Corollary 3.1}

If under the conditions of theorem $3\left|\mathfrak{a}_{i}\right|=1$, for all $i$, then $\mathcal{L}_{T}$ is ergodic.

So theorems 1.1, 1.2 and 3.1 provide a complete classification, up to the assumptions $0_{1}, \ldots, 0_{5}$.

The practical computation of ergodicity conditions can be achieved according to the following sequence :

1. Calculate the vectors of mean jumps ;

2. calculate $\operatorname{sgn} v_{\Lambda^{(1)}}$ for all ergodic $\wedge^{(1)}$, using lemma 1 ;

3. calculate the scattering probabilities, using formulas $(2.2)-(2.4)$;

4. calculate the stationary probabilities of the associated chain, which in the general case give rise to a system of $|T|$ linear equations ;

5. use theorems 1 to 3 . 


\section{Proof of Ergodicity in Theorem 3}

Let us first consider the case when there is one essential class with at least 2 essential states. So, all 1-dimensional faces are not ergodic. We introduce the following Markov process $\eta_{t}$ on $\tilde{T}$ : a particle moves along the constant vector field $M_{\Lambda^{(2)}}$ (with velocity $M_{\Lambda^{(2)}}$ ) on any face $\tilde{\Lambda}^{(2)} \subset \tilde{T}$. When it reaches a 1-dimensional face $\tilde{\Lambda}^{(1)}$, it chooses with probability $p\left(\wedge^{(1)}, \wedge_{i}^{(2)}\right)$ a face $\wedge_{i}^{(2)} \in S_{-}\left(\wedge^{(1)}\right)$ and continues its way along $\tilde{\Lambda}_{i}^{(2)}$ and so on.

The process $\eta_{t}$ is called ergodic if the mean time $f_{x}$ of reaching 0 starting from a point $x \in \tilde{T}$ is finite (for all $x$ ).

Lemma $4.1 \eta_{t}$ is ergodic if (3.2) holds.

Proof: It is a direct consequence of the following fact : if $x$ is far enough from 0 , then until reaching $0, \eta_{t}$ visits a 2-dimensional face $\wedge^{(2)}$ with a frequency exponentially close to $\pi\left(\wedge^{(2)}\right)$. (So $\mathfrak{A}$ is the induced chain for $\eta_{t}$ in some sense).

We should like (as in [1]) to use $f_{x}$ as a Lyapounov function for $\mathcal{L}_{T}$ in the following criterion for ergodicity [1] :

$\mathcal{L}_{T}$ is ergodic iff there exists a positive integer valued function $m(\alpha)$ and $\epsilon>0$ such that

$$
\sum_{\beta} \tilde{p}_{\alpha \beta}^{(m(\alpha))} f_{\beta}-f_{\alpha}<-\epsilon m(\alpha),
$$

for $\alpha \in T-T_{0}$ and some finite set $T_{0} \subset T$. Let $\hat{p}_{\alpha \beta}^{(t)}$ be the transition probabilities in $t$ steps of the process $\eta_{t}$ for $\alpha, \beta \in \tilde{T}$. For $\alpha$ and $t$ given, they differ from zero only for a finite number of points $\beta$.

Let us suppose $M<0$. Then $\eta_{t}$ is ergodic and so, for $\alpha$ not very close to the origin,

$$
f_{\alpha}=1+\sum_{\beta} \tilde{p}_{\alpha \beta}^{(1)} f_{\beta}
$$

or

$$
\sum_{\beta} \tilde{p}_{\alpha \beta}^{(1)} f_{\beta}-f_{\alpha}=-1
$$


Let us note that $f_{\alpha}$ has the following properties :

1. It is continuous everywhere except on the 1-dimensional chains where scattering occurs ;

2. $C_{1}|x| \leq f_{x} \leq C_{2}|x|$, for some $C_{1}, C_{2}>0$;

3. For any 2-dimensional face $\wedge^{(2)}$, the function $f_{x}$ has a linear decrease along any line parallel to $M_{\wedge^{(2)}}$ in the direction of $M_{\wedge^{(2)}}$.

4. Due to the space homogeneity, $f_{x}$ satisfies (4.1) with $m(\alpha)=1$ everywhere, except for neighbourhoods of 1-dimensional faces. More exactly let us take some nonergodic 1-dimensional face $\tilde{\Lambda}^{(1)}$ and put for any $\tilde{\Lambda}^{(2)}$

$$
\mathcal{D}_{\wedge^{(1)}, \wedge^{(2)}}(\rho)=\left\{\alpha: \alpha \in \overline{\Lambda^{(2)}}, \rho\left(\alpha, \tilde{\Lambda}^{(1)}\right)=\rho\right\}
$$

Then, if $\wedge^{(2)} \in S_{-}\left(\wedge^{(1)}\right), \alpha \in \mathcal{D}_{\wedge^{(1)}, \wedge^{(2)}}(1),(4.1)$ can be not satisfied. For this reason, we modify our Lyapounov functions as follows. Define

$$
\tilde{f}_{\alpha}=\left\{\begin{array}{l}
f_{\alpha}, \alpha \notin \mathcal{D}\left(\rho_{0}\right), \\
\left(C_{2}+1\right)|\alpha|, \alpha \in \mathcal{D}\left(\rho_{0}\right),
\end{array}\right.
$$

where

$$
\mathcal{D}\left(\rho_{0}\right)=\bigcup_{\wedge^{(1)}} \bigcup_{\wedge^{(2)} \in S_{-}\left(\wedge^{(1)}\right)} \bigcup_{\rho=0}^{\rho_{0}} \mathcal{D}_{\wedge^{(1)}, \wedge^{(2)}}(\rho)
$$

and $\rho_{0}$ is a constant to be specified below.

Lemma 4.2 There exist $\rho_{0}, m, \delta>0$ such that (4.1) holds for the new Lyapounov function $\tilde{f}_{\alpha}$ with

$$
m(\alpha)=\left\{\begin{array}{l}
m, \quad \alpha \in \mathcal{D}\left(\rho_{0}+1\right) \\
\delta|\alpha|, \quad \alpha \in \bigcup_{\Lambda^{(1)}} \bigcup_{\Lambda^{(2)} \in S_{+}\left(\Lambda^{(1)}\right)} \mathcal{D}_{\Lambda^{(1)}, \Lambda^{(2)}}(1) \\
1, \text { in other cases }
\end{array}\right.
$$




\section{Proof}

We choose $\delta>0$ sufficiently small ; then we take $\rho_{0}$ sufficiently large and then $m=m\left(\rho_{0}\right)$ sufficiently large. When $m(\alpha)=1$, it is easy to verify (4.1), due to the linearity property 4 .

Let us now take a point $\alpha \in \mathcal{D}\left(\rho_{0}+1\right)$. One can prove that starting from $\alpha$ after $m$ steps, we shall be outside $\mathcal{D}\left(\rho_{0}\right)$ with probability $1-\epsilon_{1}$, where $\epsilon_{1}=\epsilon_{1}(m) \rightarrow 0$ when $m \rightarrow \infty$, uniformly in $\alpha$, with $|\alpha|>a_{0}$, for some $a_{0}$ sufficiently large. This follows just from the transience of the corresponding hedgehog.

It follows that (4.1) holds since, for large $m$, we can take $\epsilon_{1}$ arbitrarily small with

$$
\tilde{f}(\beta)=f(\beta)<C_{2}(|\alpha|+m d),
$$

where $\beta \notin \mathcal{D}\left(\rho_{0}\right)$ is the final point after $m$ steps.

Let now $\alpha \in \mathcal{D}_{\Lambda^{(1)}, \wedge^{(2)}}(1), \quad \wedge^{(2)} \in S_{+}\left(\wedge^{(1)}\right)$ and $\xi_{t}(\alpha)$ be the position of the random walk starting from $\alpha$.

Let $\wedge_{i} \in S_{-}\left(\wedge^{\prime}\right)$. Let us denote $\eta_{t}\left(\alpha, \wedge_{i}\right)$ the point of $\mathbf{R}_{+}^{2}=\wedge_{i}$ which is the unique point of $\Lambda_{i}$, where process $\eta_{t}$ is to be found at time $t$, after having started from $\alpha$.

Lemma 4.3 Let $t=\delta|\alpha|$. Then, for any $\epsilon_{2}, \epsilon_{3}>0$ sufficiently small, there exists $a_{0}>0$ such that, for any $|\alpha|>a_{0}$ and for any $\wedge_{i} \in S_{-}\left(\wedge^{(1)}\right)$,

$$
\left|\operatorname{Prob}\left\{\xi_{t}(\alpha) \in \wedge_{i},\left|\xi_{t}(\alpha)-\eta_{t}\left(\alpha, \wedge_{i}\right)\right|<\epsilon_{3}|\alpha|\right\}-p\left(\wedge, \wedge_{i}\right)\right|<\frac{\epsilon_{2}}{l},
$$

where $l=\left|S_{-}\left(\wedge^{(1)}\right)\right|$.

\section{Proof}

From the point $\alpha \in \mathcal{D}_{\wedge^{\prime}, \wedge}(1)$, we make first $\frac{\epsilon_{3}}{2(d+1)}|\alpha|$ jumps. Then, for $|\alpha|$ large enough, with the probability $p_{i}$ such that

$$
\left|p_{i}-p\left(\wedge, \wedge_{i}\right)\right|<\frac{\epsilon_{2}}{3 l},
$$

we shall be in some point $\alpha_{i} \in \wedge_{i}$ satisfying 


$$
\rho\left(\alpha_{i}, \eta_{t}\left(\alpha, \wedge_{i}\right)\right)<\frac{1}{2} \epsilon_{3}|\alpha|
$$

After starting from $\alpha_{i}$, we perform the remaining $\left(\delta-\frac{\epsilon_{3}}{2(d+1)}\right)|\alpha|$ jumps. This will be in fact a translation invariant random walk in $\mathbf{Z}^{2}$ and, using Kolmogorov's inequality, we prove that, for $|\alpha|$ large, it will never go out of $\wedge_{i}$ with probability $1-\frac{\epsilon_{2}}{3}$.

But, moreover, by the Law of Large Numbers, its final point $\xi_{t}\left(\alpha_{i}, \alpha\right)$ will satisfy the inequality

$$
\xi_{t}\left(\alpha_{i}, \alpha\right)-\left(\alpha_{i}+M_{\wedge_{i}}\left(\delta-\frac{\epsilon_{3}}{2(d+1)}\right)|\alpha|\right)<\frac{1}{2} \epsilon_{3}|\alpha|,
$$

with probability $1-\frac{\epsilon_{2}}{3 l}$.

Putting together all these estimates, we get (4.3), concluding the proof of lemma 4.3.

We can finish now the proof of lemma 4.2.

From (4.2), we have

$$
\sum_{\beta} \tilde{p}_{\alpha \beta}^{(t)} \tilde{f}_{\beta}-\tilde{f}_{\alpha}=-t
$$

Comparing (4.1) and (4.4) yields

$$
\sum p_{\alpha \beta}^{(t)} \tilde{f}_{\beta}-\tilde{f}_{\alpha}=\sum_{\beta} \tilde{p}_{\alpha \beta}^{(t)} \tilde{f}_{\beta}-\tilde{f}_{\alpha}+\Delta
$$

where

$$
\Delta<\epsilon_{2}\left(C_{2}+1\right)|\alpha|(1+d \delta)+\epsilon_{3}|\alpha| .
$$

So for $\epsilon_{2}, \epsilon_{3}$ small we get

$$
\sum p_{\alpha \beta}^{(t)} \tilde{f}_{\beta}-\tilde{f}_{\alpha}<-\frac{1}{2} \delta|\alpha|,
$$

Concluding the proof of lemma 4.2 and the ergodicity part of theorem 3.1 . 
If there are several essential classes, we use the same Lyapounov function as before inside 2-dimensional faces and in a vicinity of nonergodic 1-dimensional faces. We define it in a neighbourhood of ergodic faces, exactly as it was done in [1] for $\mathbf{Z}_{+}^{3}$.

\section{Proof of the transience}

First we shall terminate the proof of theorem 3.1 .

Assume that, for the class $\mathfrak{A}_{i}$,

$$
M\left(\mathfrak{a}_{i}\right)>0 .
$$

We shall prove then that $\mathcal{L}_{T}$ is transient. It will be, for notational convenience, more convenient to consider the modified chain $\check{\mathcal{L}}_{T}$ instead of $\mathcal{L}_{T}$, with the following transition probabilities

$$
\begin{gathered}
\check{p}_{\alpha \beta}=p_{\alpha \beta}, \text { if } \alpha \neq \underline{0}, \\
\check{p}_{00}=1, \check{p}_{0 \beta}=0, \beta \neq \underline{0} .
\end{gathered}
$$

Thus it is sufficient to prove the non recurrence of $\mathcal{L}$.

Let $\dot{\xi}_{t}(\alpha)$ be the position of the random walk corresponding to $\dot{\mathcal{L}}$, corresponding to the initial condition $\alpha$, i.e. $\check{\xi}_{0}(\alpha)=\alpha$.

Choosing $\alpha \neq \underline{0}$ belonging to some $\wedge^{(1)}$, we define the following a sequence of random times $0=\tau_{0}<\tau_{1}<\ldots<\tau_{n}<\ldots$ by induction : $\tau_{n}$ is the first after $\tau_{n-1}$ hitting time of $\underline{0}$ or some 1-dimensional face $\wedge^{(1)}=\wedge^{(1)}$ different from the face $\wedge_{n-1}^{(1)}$ to which $\check{\xi}_{\tau_{n-1}}(\alpha)$ belongs. Of course, if $\check{\xi}_{\tau_{n}}(\alpha)=0$ for some $\tau_{n}$, then $\check{\tau}_{m}=\tau_{n}$, for $m \geq n$.

Let us consider the new Markov chain $\zeta_{n}(\alpha)=\check{\xi}_{\tau_{n}}(\alpha), \zeta_{0}(\alpha)=\alpha$, the state space of which is the union of all 1-dimensional faces $U \wedge^{(1)}$ and $\underline{0}$. The probability of sometimes hitting $\underline{0}$ are equal for $\xi_{n}(\alpha)$ and $\zeta_{n}(\alpha)$. So it is sufficient to prove the non-recurrence of $\zeta_{n}(\alpha)$.

Lemma 5.1 Let us consider two 1-dimensional faces $\wedge^{(1)}, \wedge_{1}^{(1)}$, and the corresponding 2-dimensional ones 


$$
\wedge_{1}^{(2)} \in S_{+}\left(\wedge^{(1)}\right), \wedge^{(2)} \in S_{-}\left(\wedge^{(1)}\right) \bigcap S_{+}\left(\wedge_{1}^{(1)}\right)
$$

Then, for any $\epsilon>0$, one can find $D_{1}>0$ such that, for any $\beta \in \wedge^{(1)}$,

$$
\left|P\left\{\zeta_{1}(\beta) \in \wedge_{1}^{(1)},\left|\zeta_{1}(\beta)\right| \leq|\beta|\left(\operatorname{tg} \phi_{\wedge_{1}^{(2)}}-\epsilon\right)\right\}-p\left(\wedge_{1}^{(2)}, \wedge^{(2)}\right)\right| \leq \frac{D_{1}}{|\beta|}
$$

\section{Proof}

Let us note first that, for any $\wedge_{2}^{(2)} \in S_{+}\left(\wedge^{(1)}\right)$, we have $p\left(\wedge_{1}^{(2)}, \wedge^{(2)}\right)=p\left(\wedge_{2}^{(2)}, \wedge^{(2)}\right)$. As the jumps are bounded, we have $\tau_{1} \geq\left[\frac{\mid \beta\rfloor}{d}\right]$ a.s. It follows (see lemma $1.1[1]$ ) that there exist $\delta_{1}, \delta_{2}, D_{2}>0$, such that, for any $\beta \in \wedge^{(1)}$ and any $t \in \mathbf{Z}_{+}, t<\left[\frac{\lfloor\beta\rfloor}{d}\right]$,

$$
P\left\{\rho\left(\check{\xi}_{t}(\beta), \wedge^{(1)}\right)<\delta_{1} t\right\} \leq D_{2} e^{-\delta_{2} t}
$$

We also have

$$
\left|P\left\{\rho\left(\check{\xi}_{t}(\beta), \wedge^{(1)}\right)>\delta_{1} t, \check{\xi}_{t}(\beta) \in \wedge^{(2)}\right\}-p\left(\wedge_{1}^{(2)}, \wedge^{(2)}\right)\right| \leq C_{3} e^{-\delta_{2} t},
$$

for any $t<\frac{|\beta|}{d}$, where $C_{3}$ is a positive constant.

For any $\epsilon_{1}, 0<\epsilon_{1}<1$, and $t_{1}=\left[\epsilon_{1} \frac{|\beta|}{d}\right]$,

$$
\left|\check{\xi}_{t_{1}}(\beta)-\beta\right| \leq \epsilon_{1}|\beta| \text {. }
$$

To prove (5.2), it is sufficient by $(5.3),(5.4)$ to prove that, for $\dot{\xi}_{t_{1}}(\beta)$ such that

$$
\rho\left(\check{\xi}_{t_{1}}(\beta), \wedge^{(1)}\right)>\delta_{1} t_{1}
$$

and for any $\epsilon_{2}>0$, there exists a constant $C_{4}=C_{4}\left(\epsilon_{1}, \epsilon_{2}\right)>0$ (not depending on $\beta$ ) such that

$$
P\left\{\check{\xi}_{\tau_{1}}(\beta) \in \wedge_{1}^{(1)}, \rho\left(\dot{\xi}_{\tau_{1}}(\beta), \check{\xi}_{t_{1}}(\beta)+\left(\tau_{1}-t_{1}\right) M_{\wedge^{(2)}}\right)>\epsilon_{2}|\beta| / \rho\left(\check{\xi}_{t_{1}}(\beta), \wedge^{(1)}\right)>\delta_{1} t_{1}\right\} \leq \frac{C_{4}}{\beta} .
$$


To prove (5.5), we consider $\wedge^{(2)}$ imbedded into $\mathbf{Z}^{2}$ and the space homogeneous r.w. $\xi_{t}^{\prime}, t \in \mathbf{Z}_{+}$, on this $\mathbf{Z}^{2}$, with initial value

$$
\xi_{0}^{\prime}=\check{\xi}_{t_{1}}(\beta)
$$

and one step transition probabilities

$$
p^{\prime}(\alpha, \beta)=p^{\prime}\left(\alpha+\alpha^{\prime}, \beta+\beta^{\prime}\right)=p(\alpha, \beta),
$$

for all $\alpha, \beta \in \wedge^{(2)}, \alpha^{\prime}, \beta^{\prime} \in \mathbf{Z}^{2}$.

Let $\tau^{\prime}$ be the first hitting time $\Lambda_{1}^{(1)}$ by $\xi_{t}^{\prime}$. We can choose $T \in \mathbf{Z}_{+}$and $\epsilon_{3}>0$, so that

$$
\rho\left(\xi_{0}^{\prime}, \wedge^{(1)}\right) \geq \delta_{1} t=\delta_{1}\left[\epsilon_{1} \frac{|\beta|}{d}\right]>\epsilon_{3}|\beta|
$$

and

$$
\begin{gathered}
\rho\left(\xi_{0}^{\prime}, \wedge_{1}^{(1)}\right)+T P r_{\wedge^{(1)}} M_{\wedge^{(2)}}+\epsilon_{3}|\beta| \\
\leq|\beta|\left(1+\epsilon_{1}\right)+T P r_{\wedge^{(1)}} M_{\wedge^{(2)}}+\epsilon_{3}|\beta|<0 .
\end{gathered}
$$

Let us note that, if

$$
\max _{t \in[0, T]}\left|\xi_{t}^{\prime}-\xi_{0}^{\prime}-M_{\wedge(2)} t\right| \leq \epsilon_{3}|\beta|,
$$

then $\tau^{\prime}<T$ and $\xi_{t}^{\prime} \in \wedge^{(2)}$ for all $t<\tau^{\prime}$.

From this remark and Kolmogorov's inequality, we get

$$
\begin{gathered}
P\left\{\check{\xi}_{\tau_{1}}(\beta) \in \wedge_{1}^{(1)}, \rho\left(\check{\xi}_{\tau_{1}}(\beta), \beta_{1}+M_{\wedge^{(2)}}\left(\tau_{1}-t_{1}\right)\right) \leq \epsilon_{3}|\beta| / \check{\xi}_{t_{1}}(\beta)\right\} \\
\geq P\left\{\max _{t \in\left[t_{1}, \tau_{1}\right]}\left|\check{\xi}_{t}(\beta)-\check{\xi}_{t_{1}}(\beta)-\left(t-t_{1}\right) M_{\Lambda^{(2)}}\right| \leq \epsilon_{3}|\beta| / \check{\xi}_{t_{1}}(\beta)\right\}= \\
=P\left\{\max _{t \in\left[0, \tau^{\prime}\right]}\left|\xi_{t}^{\prime}-\xi_{0}-t M_{\wedge(2)}\right| \leq \epsilon_{3}|\beta|\right\}
\end{gathered}
$$




$$
\begin{gathered}
\geq P\left\{\max _{t \in[0, T]}\left|\xi_{t}^{\prime}-\xi_{0}-t M_{\wedge(2)}\right| \leq \epsilon_{3}|\beta|\right\} \\
\geq 1-\frac{C_{5} T}{\epsilon_{3}^{2}|\beta|^{2}},
\end{gathered}
$$

for some constant $C_{5}>0$.

The last step to derive (5.5) is now achieved by choosing $T$ such that $T \leq$ const $|\beta|$. Lemma 5.1 is proved.

It follows from 5.1 that, for any sequence $\wedge_{0}, \ldots, \wedge_{n} \in \mathfrak{x}_{i}$ of 2-dimensional faces, for any $\alpha \in \bar{\Lambda}_{0} \cap \bar{\Lambda}_{1}$ and any $\epsilon>0, n \in \mathbf{Z}_{+}$, there exist $C_{6}=C_{6}(\epsilon, n)$ not depending on $\alpha \in \pi_{0} \cap \pi_{1}$, such that.

$$
\begin{gathered}
\mid P\left\{\zeta_{k}(\alpha) \in \bar{\wedge}_{k} \bigcap \bar{\Lambda}_{k+1},\left|\zeta_{k}(\alpha)\right| \geq\left(\operatorname{tg} \phi_{\wedge_{k}}-\epsilon\right)\left|\zeta_{k-1}(\alpha)\right|, k=1, \ldots, n\right\} \\
-p\left(\wedge_{0}, \wedge_{1}\right) p\left(\wedge_{1}, \wedge_{2}\right) \ldots p\left(\wedge_{n-1}, \wedge_{n}\right) \mid \leq \frac{C_{6}}{|\alpha|}
\end{gathered}
$$

Let us choose $\epsilon>0, \delta>0$ and $n \in \mathbf{Z}_{+}$so that, for any $\wedge_{0} \in \mathfrak{A}_{i}$,

$$
p^{(n)}\left(\wedge_{0}, \wedge_{0}\right)>0
$$

and

$$
\sum_{k=1}^{n} \sum_{\wedge^{(2)}} p^{(k)}\left(\wedge_{0}, \wedge^{(2)}\right) \log \left(\operatorname{tg} \phi_{\wedge^{(2)}}-\epsilon\right)>\delta>0 .
$$

Due to $(5.1)$, it is always possible to satisfy (5.7) since

$$
\frac{1}{n} \sum_{k=1}^{n} p^{(k)}\left(\wedge_{0}, \wedge^{(2)}\right) \rightarrow \pi\left(\wedge^{(2)}\right)
$$

for any $\wedge_{0}, \wedge^{(2)}$, whenever $\wedge_{0}$ is an essential state in the associated Markov chain.

To prove the transience of $\zeta_{k}(\alpha), k \in \mathbf{Z}_{+}$, it is sufficient to prove the transience of the chain $\eta_{k}=\xi_{n k}(\alpha), k \in \mathbf{Z}_{+}$. We shall use Foster's critcrion 
[] recalled thereafter : if there exists a positive function $f$ defined on the state space $X$ and a set $A \subset X$, such that

$$
\text { and }\left\{\begin{array}{l}
\sum_{j \in X} p_{i j} f_{j} \leq f_{i}, \forall i \in X-A, \\
\inf _{i \in A} f_{i}>\sup _{j \in X-A} f_{j}
\end{array}\right.
$$

then the Markov chain is transient.

We define $f$ on the state space of $\eta_{k}$.

$$
f(\alpha)=\left\{\begin{array}{l}
\frac{1}{\log 3|\alpha|}, \text { if } \alpha \neq 0 \text { and } \alpha \in \pi^{(2)}, \text { for some } \wedge^{(2)} \in \mathfrak{A}_{i}, \\
1, \text { in the other cases. }
\end{array}\right.
$$

Let us prove that, if $\left|\eta_{0}\right|$ is sufficiently large, then

$$
E\left(f\left(\eta_{1}\right)\right) \leq f\left(\eta_{0}\right) .
$$

In fact, if $f\left(\eta_{0}\right)=1$, then (5.8) evidently holds. Let

$$
f\left(\eta_{0}\right)=\frac{1}{\log 3\left|\eta_{0}\right|} \text { and } \eta_{0} \in \Lambda^{(1)}
$$

for some 1-dimensional face $\wedge^{(1)}$. Then, by (5.6),

$$
E\left(f\left(\eta_{1}\right)\right) \leq \sum_{\wedge_{1}, \ldots, \wedge_{n}} p\left(\wedge_{0}, \wedge_{1}\right) \ldots p\left(\wedge_{n-1}, \wedge_{n}\right) \cdot \frac{1}{\log \left[3\left|\eta_{0}\right| \prod_{j=1}^{n}\left(\operatorname{tg} \phi_{\wedge_{j}}-\epsilon\right)\right]}+\frac{C_{6}}{\left|\eta_{0}\right|}
$$

where $\wedge_{0} \in S_{-}\left(\wedge^{(1)}\right), \wedge_{0} \in \mathfrak{a}_{i}$.

But, for $\left|\eta_{0}\right|$ sufficiently large,

$$
\begin{gathered}
\left(\log \left[3\left|\eta_{0}\right| \prod_{j=1}^{n}\left(\operatorname{tg} \phi_{\Lambda_{j}}-\epsilon\right)\right]\right)^{-1}= \\
=\frac{1}{\log 3\left|\eta_{0}\right|}\left[1+\frac{1}{\log 3\left|\eta_{0}\right|}\left(\sum_{j=1}^{n} \log \left(\operatorname{tg} \phi_{\Lambda_{j}}-\epsilon\right)\right)\right]^{-1} \leq
\end{gathered}
$$




$$
\leq \frac{1}{\log 3\left|\eta_{0}\right|}-\frac{\sum_{j=1}^{n} \log \left(\operatorname{tg} \phi_{\Lambda}-\epsilon\right)}{\left(\log 3\left|\eta_{0}\right|\right)^{2}}+o\left(\frac{1}{\left(\log 3\left|\eta_{0}\right|\right)^{2}}\right)
$$

From (5.9), (5.10), (5.7), we get

$$
E\left(f\left(\eta_{1}\right)\right) \leq \frac{C_{6}}{\left|\eta_{0}\right|}+\frac{1}{\log 3\left|\eta_{0}\right|}-\frac{\delta}{\left(\log 3\left|\eta_{0}\right|\right)^{2}}+o\left(\frac{1}{\left(\log 3\left|\eta_{0}\right|\right)^{2}}\right)
$$

Theorem 3.1 is proved.

Theorems 1.1 and 1.2 are in fact implicity contained in the results of [1]. We shall briefly explain how they could be proved.

The proof of theorem 1.1 is based on the following criterion of transience :

Lemma 5.2 Assume that, for the irreducible Markov chain with state space $S$, there exists a function $f: S \rightarrow R_{+}$, such that

(i) for some $d>0$,

$\left|f_{\alpha}-f_{\beta}\right|>d \Longrightarrow p_{\alpha \beta}=0, \alpha, \beta \in S$;

(ii) $\sum_{\beta} p_{\alpha \beta} f_{\beta}-f_{\alpha} \geq \epsilon$, for some $\epsilon>0$ and all $\alpha \in A_{C}=\left\{\alpha: f_{\alpha}>C\right\} \neq \emptyset$ , for some $C>0$.

Then the chain is transient.

Proof : See [1].

Let $\wedge^{(2)}$ be a two-dimensional face, with $M_{\wedge^{(2)}}$ having both components positive. Then we can construct a Lyapounov function $f$, taking it equal to 0 outside $\wedge^{(2)}$ and $\epsilon$-linear on $\wedge^{(2)}$, as in [2].

To prove theorem 1.2, we could use condition $B^{\prime}$ and theorem 2.1 of [1].

\section{Remarks and problems}

\section{Remark 1}

In our case, we could have defined an associated Markov chain with a different set of states : $\left\{\wedge^{(1)}\right\}$ instead of $\left\{\wedge^{(2)}\right\}$ with transition probabilities

$$
p\left(\wedge^{(1)}, \wedge_{1}^{(1)}\right)=p\left(\wedge^{(2)}, \wedge_{1}^{(2)}\right)
$$


for any $\wedge^{(2)} \in S_{+}\left(\wedge^{(1)}\right)$ and the unique $\wedge_{1}^{(2)} \in S_{+}\left(\wedge_{1}^{(1)}\right) \cap S_{-}\left(\wedge^{(1)}\right)$, if such $\wedge_{1}^{(2)}$ exists and 0 otherwise: In the more general case when condition 1a) of boundedness of jumps does not take place, the situation becomes more complicated : the scattering probabilities depend on the ingoing bristle of the hedgehog. Thus, the probabilities $p\left(\wedge^{(2)}, \wedge_{1}^{(2)}\right)$ and $p\left(\wedge_{2}^{(2)}, \wedge_{1}^{(2)}\right)$ can be different for different $\wedge^{(2)}, \wedge_{2}^{(2)} \in S_{+}\left(\wedge^{(1)}\right)$. But this case can be treated as well by the same methods.

\section{Remark 2}

It is of interest to generalize our results to the case when the simplexes of our complex are not $\mathbf{Z}_{+}^{2}$, but angles in $R_{+}^{2}$ or in $\mathbf{Z}_{+}^{(2)}$. Of special interest is the situation when these angle in $Z_{+}^{2}$ are not commensurable with $\pi$.

\section{Remark 3}

Our methods allow to get the classification of random walks in $\mathbf{Z}_{+}^{N}$ under the same "non zero" and homogeneity assumptions, when all vectors of mean jumps inside all faces $\wedge$ with $\operatorname{dim} \wedge \geq 3$ have their coordinates negative. Then $M_{\wedge}$, with $\operatorname{dim} \wedge=1$ or 2 , will become vectors derived from the corresponding induced chains.

\section{Remark 4}

We want to show now that all our assumptions have Lebesgue measure 1 in the parameter space. $0_{1}, 0_{3}$ are fulfilled when all the $p_{\alpha \beta}$ 's are positive. $0_{2}$ is satisfied except a for finite number of hyperplanes. $0_{4}, 0_{5}$ are not fulfilled only when $V_{\wedge}(1)$ in lemma 1.3 is equal to zero. 


\section{References}

1. V.A. Malyshev, M.V. Menshikov. "Ergodicity, continuity and analyticity of countable Markov chains". Transactions of Moscow Math. Soc. 39 (1979).

2. V.A. Malyshev. "Classification of two-dimensional positive random walks and almost linear semi-martingales". Dokl. Akad. Nauk USSR, 202 (1972), 526-528.

3. M.V. Menshikov. "Ergodicity and transience conditions for random walks in the positive octant of space". Dokl. Ac. Sc. USSR, 217 (1974), 755-758. 



\section{ISSN 0249-6399}

